\title{
DISPERSION OF MAGNETIC FIELDS IN MOLECULAR CLOUDS. IV. ANALYSIS OF INTERFEROMETRY DATA
}

\author{
Martin Houde ${ }^{1,2}$, Charles L. H. Hull ${ }^{3,8}$, Richard L. Plambeck ${ }^{4}$, John E. Vaillancourt ${ }^{5}$, and Roger H. Hildebrand ${ }^{6,7}$ \\ ${ }^{1}$ Department of Physics and Astronomy, The University of Western Ontario, London, ON N6A 3K7, Canada \\ ${ }^{2}$ Division of Physics, Mathematics and Astronomy, California Institute of Technology, Pasadena, CA 91125, USA \\ ${ }^{3}$ Harvard-Smithsonian Center for Astrophysics, 60 Garden St., Cambridge, MA 02138, USA \\ ${ }^{4}$ Astronomy Department \& Radio Astronomy Laboratory, University of California, Berkeley, CA 94720-3411, USA \\ ${ }^{5}$ Stratospheric Observatory for Infrared Astronomy, Universities Space Research Association, NASA Ames Research Center, Moffet Field, CA 94035, USA \\ ${ }^{6}$ Department of Astronomy and Astrophysics and Enrico Fermi Institute, The University of Chicago, Chicago, IL 60637, USA \\ ${ }^{7}$ Department of Physics, The University of Chicago, Chicago, IL 60637, USA \\ Received 2015 November 25; accepted 2016 February 4; published 2016 March 16
}

\begin{abstract}
We expand on the dispersion analysis of polarimetry maps toward applications to interferometry data. We show how the filtering of low spatial frequencies can be accounted for within the idealized Gaussian turbulence model, initially introduced for single-dish data analysis, to recover reliable estimates for correlation lengths of magnetized turbulence, as well as magnetic field strengths (plane-of-the-sky component) using the Davis-ChandrasekharFermi method. We apply our updated technique to TADPOL/CARMA data obtained on W3 $(\mathrm{OH})$, W3 Main, and DR21(OH). For W3(OH), our analysis yields a turbulence correlation length $\delta \simeq 19 \mathrm{mpc}$, a ratio of turbulent-tototal magnetic energy $\left\langle B_{\mathrm{t}}^{2}\right\rangle /\left\langle B^{2}\right\rangle \simeq 0.58$, and a magnetic field strength $B_{0} \sim 1.1 \mathrm{mG}$; for W3 Main $\delta \simeq 22 \mathrm{mpc}$, $\left\langle B_{\mathrm{t}}^{2}\right\rangle /\left\langle B^{2}\right\rangle \simeq 0.74$, and $B_{0} \sim 0.7 \mathrm{mG}$; while for DR21(OH) $\delta \simeq 12 \mathrm{mpc},\left\langle B_{\mathrm{t}}^{2}\right\rangle /\left\langle B^{2}\right\rangle \simeq 0.70$, and $B_{0} \sim 1.2 \mathrm{mG}$.
\end{abstract}

Key words: ISM: clouds - ISM: magnetic fields - polarization - turbulence

\section{INTRODUCTION}

Given the difficulties of directly measuring magnetic fields in the interstellar medium (ISM), with Zeeman observations still being the only means for achieving this goal (Heiles 1997; Crutcher et al. 1999; Falgarone et al. 2008), plane of sky linear polarization maps have become in the last few decades the primary way by which magnetic field studies have been pushed forward. Qualitative analyses of magnetic fields morphologies from polarization maps and data have recently been replaced by more quantitative techniques to provide a better view and understanding of magnetized turbulence in the ISM. Although structure functions developed for the studies of turbulence in general (Frisch 1995) had been previously used with polarization maps to study large-scale behavior in the orientation of magnetic fields (Kobulnicky et al. 1994; Dotson 1996) or of polarized intensities (Beck et al. 1999), more recent works have introduced novel methods aimed at studying magnetized turbulence on smaller scales (Falceta-Gonçalves et al. 2008; Hildebrand et al. 2009; Houde et al. 2009, 2011).

Following the study of Falceta-Gonçalves et al. (2008), who applied the structure function of the polarization angle to simulations, Hildebrand et al. (2009) (hereafter Paper I) applied the technique to actual data obtained with the Hertz polarimeter (Dowell et al. 1998) to generally address one issue that had been a source of error when such data were used with the socalled Davis-Chandrasekhar-Fermi (DCF) method (Davis 1951; Chandrasekhar \& Fermi 1953). That is, in Paper I, among other things, a method based on the expected difference in length scales between the turbulent and ordered (or large-scale) components of the magnetic field was introduced to remove the latter's unwanted contribution to the angular dispersion used in the DCF equation, without

\footnotetext{
8 Jansky Fellow of the National Radio Astronomy Observatory, which is a facility of the National Science Foundation operated under cooperative agreement by Associated Universities, Inc.
}

having to assume any shape for the ordered field orientation. A second issue that also affected estimates of magnetic field strengths with the DCF method is the unavoidable signal integration across the telescope beam and through the depth of the sources probed by the observations. This issue, first discussed by Myers \& Goodman (1991) for the case of a narrow, pencil-like telescope beam, brings a systematic decrease of the apparent level of turbulence (or the angular dispersion measured from the polarization pseudo-vectors) and a corresponding erroneous increase in the field strength obtained with the DCF equation. Houde et al. (2009) (hereafter Paper II) showed how this could be properly handled for single-dish observations by developing an analytical solution for the problem using an isotropic Gaussian turbulence model. This not only allowed to correct for the signal integration problem, but also made it possible to provide estimates for magnetized turbulence correlation lengths. For example, from OMC-1 SHARP data (Novak et al. 2004; Li et al. 2006, 2008), they measured a turbulent correlation length of $16 \mathrm{mpc}$ and a magnetic field strength of approximately $760 \mu \mathrm{G}$. The analysis developed in Papers I and II were since applied in several studies led by different teams of researchers (see, for example, Franco et al. 2010; Chapman et al. 2011; Girart et al. 2013; Planck Collaboration Int. XXXV 2016).

Subsequently, the dispersion analysis was further developed and applied to studies of the magnetized turbulent power spectrum and the potential determination of turbulence dissipation scales (Houde et al. 2011, hereafter Paper III), and magnetohydrodynamics (MHD) turbulence anisotropy in molecular clouds (Chitsazzadeh et al. 2012, hereafter Paper IV). Eventually the Gaussian turbulence model was extended and solved for the more general case of twodimensional turbulence and successfully applied to the synchrotron polarization data of M51 from Fletcher et al. (2011) to clearly reveal the anisotropy in the turbulent component of the magnetic field (expected from MHD 
Table 1

Glossary

\begin{tabular}{|c|c|c|}
\hline Parameter & Definition & Reference \\
\hline$a$ & Integration variable-vector position on the plane of the sky (POS) & Equations (2), (9) \\
\hline$a_{2 j}$ & Taylor expansion coefficients for $\alpha^{2}(\ell)$ & Equation (8) \\
\hline$B(x)$ & Total three-dimensional magnetic field & Equation (4) \\
\hline$B_{\mathrm{t}}(\boldsymbol{x})$ & Three-dimensional turbulent magnetic field & Equation (4) \\
\hline $\bar{B}(\boldsymbol{r})$ & Total integrated POS magnetic field & Equation (2) \\
\hline $\bar{B}_{0}(\boldsymbol{r})$ & Integrated POS ordered magnetic field & Equation (6) \\
\hline $\boldsymbol{e}_{r}$ & Unit vector along $r$ on the POS & Equation (3) \\
\hline $\boldsymbol{e}_{z}$ & Unit vector along the line of sight & Equation (3) \\
\hline$F_{0}(\boldsymbol{a}, z)$ & Ordered polarized emission & Equation (2) \\
\hline$H(\boldsymbol{r})$ & Telescope beam profile & Equations (2), (9), (12), (18) \\
\hline$H\left(\boldsymbol{k}_{v}\right)$ & Fourier transform of telescope beam profile & Equation (10) \\
\hline$\ell$ & Distance between measurement pairs (POS) & Section 2 \\
\hline $\mathcal{R}_{3 \mathrm{D}, \mathrm{t}}\left(\boldsymbol{k}_{v}, k_{u}\right)$ & Power spectrum of the intrinsic three-dimensional magnetized turbulence & Equation (10) \\
\hline $\mathcal{R}_{\mathrm{t}}\left(\boldsymbol{k}_{v}\right)$ & Power spectrum of integrated two-dimensional magnetized turbulence & Equation $(10)$ \\
\hline$W_{1}, W_{2}$ & Gaussian telescope beam radii (standard deviation equivalent) & Equations (12), (18) \\
\hline $\boldsymbol{x}=r \boldsymbol{e}_{r}+z \boldsymbol{e}_{z}$ & Three-dimensional position vector & Equation (3) \\
\hline$z$ & Position along the line of sight & Equation (3) \\
\hline$\alpha^{2}(\ell)$ & Normalized autocorrelation of the integrated ordered POS magnetic field & Equation (6) \\
\hline$b^{2}(\ell)$ & Normalized autocorrelation of the integrated turbulent POS magnetic field & Equations (7), (15), (23) \\
\hline$b^{2}\left(\boldsymbol{k}_{v}\right)$ & Power spectrum of the integrated turbulent POS magnetic field & Equation $(10)$ \\
\hline$\delta$ & Turbulence correlation length & Equation (11) \\
\hline$\Delta$ & Maximum depth of a molecular cloud along the line of sight & Equations (2), (9) \\
\hline$\Delta^{\prime}$ & Effective depth of a molecular cloud along the line of sight & Equations (14), (20), (21) \\
\hline$\Delta \Phi(\ell)$ & Difference in polarization angles between measurement pairs separated by $\ell$ & Section 2, Equation (1) \\
\hline
\end{tabular}

turbulence theory; Goldreich \& Sridhar 1995) in this galaxy (Houde et al. 2013, hereafter Paper V). More specific to this paper, Paper III discussed the issues that arise when the dispersion analysis is applied to high-resolution interferometry data. More precisely, the filtering of low spatial frequencies (i.e., extended structures) inherent to interferometers was shown to render questionable the application of the Gaussian turbulence model of Paper II for the dispersion analyses of such polarization data (and to the DCF method, for example).

In this paper, we revisit the application of the dispersion analysis to interferometry data by extending the isotropic Gaussian turbulence model to account for the low spatial frequency filtering process. We will specifically focus on the application of the technique and the ability to obtain reliable quantitative results rather than on the astrophysical implications of these results for the sources we will study. We start with a brief summary of the main definitions and equations for the dispersion analysis in Section 2 paying special attention to the isotropic Gaussian turbulence model as developed for singledish observations in Section 2.1, while the generalization to interferometer data is presented in Section 2.2. We then follow with an application of the new model to TADPOL/CARMA data of $\mathrm{W} 3(\mathrm{OH})$, W3 Main, and $\mathrm{DR} 21(\mathrm{OH})$ previously published by Hull et al. (2014) in Section 3, and we end with a summary and conclusion in Section 4. The details of the data processing and error propagation calculations will be found in the Appendix at the end.

\section{THE DISPERSION ANALYSIS}

As mentioned earlier, the development of the dispersion analysis, while taking into account signal integration in the column of gas subtended by the telescope beam, was initially performed in Paper II, but the first application to interferometry data with a special emphasis on the characterization of the magnetized turbulence power spectrum was done in Paper III. This model was subsequently enhanced to include anisotropic turbulence in Paper V. For convenience, we give here a brief summary of the main equations for the isotropic turbulence case and mainly focus on the Gaussian turbulence approximation introduced in Paper II for the determination of correlation lengths $\delta$ and the turbulent-to-total magnetic energy ratio $\left\langle B_{\mathrm{t}}^{2}\right\rangle /\left\langle B^{2}\right\rangle$. A glossary of the different symbols and parameters appearing in the equations of this section is given in Table 1.

Given the difference $\Delta \Phi(\ell) \equiv \Phi(\boldsymbol{r})-\Phi(\boldsymbol{r}+\boldsymbol{\ell})$ in the polarization angle $\Phi$ measured at two positions separated by a distance $\ell$ on the plane of the sky, we define the dispersion function $1-\langle\cos [\Delta \Phi(\ell)]\rangle$ for the signal-integrated magnetic field $\overline{\boldsymbol{B}}$ with

$$
\langle\cos [\Delta \Phi(\ell)]\rangle=\frac{\langle\overline{\boldsymbol{B}} \cdot \overline{\boldsymbol{B}}(\ell)\rangle}{\langle\overline{\boldsymbol{B}} \cdot \overline{\boldsymbol{B}}(0)\rangle},
$$

where $\langle\cdots\rangle$ denotes an average, $\ell=|\ell|, \quad$ and $\langle\overline{\boldsymbol{B}} \cdot \overline{\boldsymbol{B}}(\ell)\rangle \equiv\langle\overline{\boldsymbol{B}}(\boldsymbol{r}) \cdot \overline{\boldsymbol{B}}(\boldsymbol{r}+\ell)\rangle \quad$ is the autocorrelation 
function of $\overline{\boldsymbol{B}}$. The signal-integrated magnetic field is defined with

$$
\overline{\boldsymbol{B}}(\boldsymbol{r})=\iint H(\boldsymbol{r}-\boldsymbol{a})\left[\frac{1}{\Delta} \int_{0}^{\Delta} F_{0}(\boldsymbol{a}, z) \boldsymbol{B}(\boldsymbol{a}, z) d z\right] d^{2} a,
$$

where $H(\boldsymbol{r})$ is the beam profile, $\Delta$ is the maximum depth of the cloud along any line of sight, and the weighting function $F_{0}(\boldsymbol{a}, z) \geqslant 0$ scales with the (ordered) polarized emission associated with the magnetic field $\boldsymbol{B}(\boldsymbol{a}, z)$. Whether one chooses the polarized emission itself for $F_{0}$ or normalizes it beforehand is irrelevant for the analysis, as the dispersion function is based on a normalized quantity (i.e., the right-hand side of Equation (1)). Any dependency on the amplitude or units of $F_{0}$ is then removed from the analysis. The position in the cloud is given by

$$
\boldsymbol{x}=\boldsymbol{r} \boldsymbol{e}_{r}+z \boldsymbol{e}_{z}
$$

with $\boldsymbol{e}_{r}$ and $\boldsymbol{e}_{z}$ the unit basis vectors along $\boldsymbol{r}$ in the plane of the sky and the $z$-axis along the line of sight, respectively. We decompose the magnetic field $\boldsymbol{B}(\boldsymbol{x})$ into an ordered field, $\boldsymbol{B}_{0}(\boldsymbol{x})$, and a turbulent (random), zero-mean component, $\boldsymbol{B}_{\mathrm{t}}(\boldsymbol{x})$, with

$$
\boldsymbol{B}(\boldsymbol{x})=\boldsymbol{B}_{0}(\boldsymbol{x})+\boldsymbol{B}_{\mathrm{t}}(\boldsymbol{x}) .
$$

We further assumed stationarity, homogeneity, and isotropy in the magnetic field strength for Equation (1), while statistical independence between ordered and turbulent components will also be implied from now on.

Upon inserting Equations (2) and (4) into Equation (1) it is found that the latter can be expressed as the sum of turbulent and ordered terms

$$
\begin{aligned}
1-\langle\cos [\Delta \Phi(\ell)]\rangle & =\left[b^{2}(0)-b^{2}(\ell)\right]+\left[\alpha^{2}(0)-\alpha^{2}(\ell)\right] \\
& =\left\{b^{2}(0)+\left[\alpha^{2}(0)-\alpha^{2}(\ell)\right]\right\}-b^{2}(\ell),
\end{aligned}
$$

with the (signal-integrated) ordered and turbulence normalized autocorrelation functions given by

$$
\begin{aligned}
& \alpha^{2}(\ell)=\frac{\left\langle\overline{\boldsymbol{B}}_{0} \cdot \overline{\boldsymbol{B}}_{0}(\ell)\right\rangle}{\langle\overline{\boldsymbol{B}} \cdot \overline{\boldsymbol{B}}(0)\rangle} \\
& b^{2}(\ell)=\frac{\left\langle\overline{\boldsymbol{B}}_{\mathrm{t}} \cdot \overline{\boldsymbol{B}}_{\mathrm{t}}(\ell)\right\rangle}{\langle\overline{\boldsymbol{B}} \cdot \overline{\boldsymbol{B}}(0)\rangle},
\end{aligned}
$$

respectively. As the ordered function $\left[\alpha^{2}(0)-\alpha^{2}(\ell)\right]$ is expected to evolve over a much larger spatial scale than $b^{2}(\ell)$, we can expand it with a (slowly varying) Taylor series and write

$$
b^{2}(0)+\left[\alpha^{2}(0)-\alpha^{2}(\ell)\right]=b^{2}(0)+\sum_{j=1}^{\infty} a_{2 j} \ell^{2 j},
$$

where $b^{2}(0)$ is simply the turbulent-to-total magnetic energy ratio (signal-integrated). The difference in scales between the function given in Equation (8) and the signal-integrated turbulent autocorrelation function $b^{2}(\ell)$ allows for their separation and the subsequent characterization of magnetized turbulence.
Using Equation (2) the autocorrelation of the signalintegrated turbulent magnetic field can be shown to be

$$
\begin{aligned}
\left\langle\overline{\boldsymbol{B}}_{\mathrm{t}} \cdot \overline{\boldsymbol{B}}_{\mathrm{t}}(\ell)\right\rangle= & \iiint \int H(\boldsymbol{a}) H\left(\boldsymbol{a}^{\prime}+\boldsymbol{\ell}\right) \\
& \times\left[\frac{2}{\Delta} \int_{0}^{\Delta}\left(1-\frac{u}{\Delta}\right) \mathcal{R}_{3 \mathrm{D}, \mathrm{t}}(v, u) d u\right] d^{2} a^{\prime} d^{2} a,
\end{aligned}
$$

with $\mathcal{R}_{3 \mathrm{D}, \mathrm{t}}(v, u)=\left\langle F_{0}(\boldsymbol{a}, z) F_{0}\left(\boldsymbol{a}^{\prime}, z^{\prime}\right)\right\rangle\left\langle\boldsymbol{B}_{\mathrm{t}}(\boldsymbol{a}, z) \cdot \boldsymbol{B}_{\mathrm{t}}\left(\boldsymbol{a}^{\prime}, z^{\prime}\right)\right\rangle$, $u=\left|z^{\prime}-z\right|$, and $v=\left|\boldsymbol{a}^{\prime}-\boldsymbol{a}\right|$. The function $\mathcal{R}_{3 \mathrm{D}, \mathrm{t}}(v, u)$ stands for the autocorrelation of the intrinsic magnetized turbulence (i.e., unaltered by the measurement process). As was discussed in Paper III, the magnetized turbulence power spectrum is contained in the Fourier transform of $b^{2}(\ell)$ (and that of Equation (9); see Equation (7))

$$
b^{2}\left(\boldsymbol{k}_{v}\right)=\left\|H\left(\boldsymbol{k}_{v}\right)\right\|^{2} \frac{\mathcal{R}_{\mathrm{t}}\left(\boldsymbol{k}_{v}\right)}{\left\langle\bar{B}^{2}\right\rangle},
$$

where

$$
\left\langle\bar{B}^{2}\right\rangle \equiv\langle\overline{\boldsymbol{B}} \cdot \overline{\boldsymbol{B}}(0)\rangle
$$
$\mathcal{R}_{\mathrm{t}}\left(\boldsymbol{k}_{v}\right) \equiv \int \mathcal{R}_{3 \mathrm{D}, \mathrm{t}}\left(\boldsymbol{k}_{v}, k_{u}\right) \operatorname{sinc}^{2}\left(k_{u} \Delta / 2\right) d k_{u}$ is the two-dimensional turbulence power spectrum, with $\mathcal{R}_{3 \mathrm{D}, \mathrm{t}}\left(\boldsymbol{k}_{v}, k_{u}\right)$ the Fourier transform of $\mathcal{R}_{3 \mathrm{D}, \mathrm{t}}(v, u)$. Although we will not be able to achieve this in this paper, data taken at high enough spatial resolution can reveal the underlying turbulence power spectrum $\mathcal{R}_{\mathrm{t}}\left(\boldsymbol{k}_{v}\right)$ by inverting Equation (10) through the removal of the filtering due to the telescope beam (i.e., $\left\|H\left(\boldsymbol{k}_{v}\right)\right\|^{2}$, the Fourier transform of the autocorrelated beam).

\subsection{Isotropic Gaussian Turbulence Model-Single-dish}

As was shown in Papers II, IV, and V, using a Gaussian model as an idealization for isotropic magnetized turbulence leads to an analytical solution for the dispersion analysis problem when the telescope beam is also expressed as a Gaussian function. We will thus use the following expression for the magnetized turbulence autocorrelation function

$$
\mathcal{R}_{3 \mathrm{D}, \mathrm{t}}(v, u)=\left\langle F_{0}^{2}\right\rangle\left\langle B_{\mathrm{t}}^{2}\right\rangle e^{-\left(v^{2}+u^{2}\right) / 2 \delta^{2}},
$$

with $\delta$ the turbulence correlation length, $\left\langle B_{\mathrm{t}}^{2}\right\rangle=\left\langle\boldsymbol{B}_{\mathrm{t}} \cdot \boldsymbol{B}_{\mathrm{t}}(0)\right\rangle$, and $\left\langle F_{0}^{2}\right\rangle=\left\langle F_{0} F_{0}(0)\right\rangle$. The telescope beam profile of width $W_{1}$ (i.e., its standard deviation equivalent) is given by

$$
H(\boldsymbol{r})=\frac{1}{2 \pi W_{1}^{2}} e^{-r^{2} / 2 W_{1}^{2}} .
$$

Given these functions, we find the following solution for Equation (5)

$$
\begin{aligned}
1-\langle\cos [\Delta \Phi(\ell)]\rangle= & \sum_{j=1}^{\infty} a_{2 j} \ell^{2 j}+\left[\frac{1}{1+N_{1}\left\langle B_{0}^{2}\right\rangle /\left\langle B_{\mathrm{t}}^{2}\right\rangle}\right] \\
& \times\left[1-e^{-\ell^{2} / 2\left(\delta^{2}+2 W_{1}^{2}\right)}\right],
\end{aligned}
$$

with the number of turbulent cells probed by telescope beam

$$
N_{1}=\frac{\left(\delta^{2}+2 W_{1}^{2}\right) \Delta^{\prime}}{\sqrt{2 \pi} \delta^{3}}
$$

and $\Delta^{\prime}$ the effective depth of the region under study, which can be determined from the autocorrelation function of the polarized flux (see Section 3.2 of Paper II). It follows that 
data from a polarization map used to calculate the left-hand side of Equation (13) can be fitted to the model expressed on the right-hand side to provide estimates for $\delta,\left\langle B_{\mathrm{t}}^{2}\right\rangle /\left\langle B_{0}^{2}\right\rangle$, and $a_{2 j}$. The signal-integrated turbulence autocorrelation function

$$
b^{2}(\ell)=\left[\frac{1}{1+N_{1}\left\langle B_{0}^{2}\right\rangle /\left\langle B_{\mathrm{t}}^{2}\right\rangle}\right] e^{-\ell^{2} / 2\left(\delta^{2}+2 W_{1}^{2}\right)},
$$

is contained in Equation (13). We find that its width (i.e., $\sqrt{\delta^{2}+2 W_{1}^{2}}$ ) is broadened through the measurement process by the telescope beam beyond the intrinsic correlation length $\delta$ of the underlying turbulence. We also find that the "true" relative level of turbulent energy in the magnetic field is integrated down through averaging among the $N_{1}$ turbulent cells contained in the column of gas with

$$
\begin{aligned}
\frac{\left\langle B_{\mathrm{t}}^{2}\right\rangle}{\left\langle B_{0}^{2}\right\rangle} & =N_{1}\left[\frac{b^{2}(0)}{1-b^{2}(0)}\right] \\
& \simeq N_{1} b^{2}(0),
\end{aligned}
$$

with the last equation valid when $b^{2}(0) \ll 1$.

An example of a hypothetical dispersion analysis for the idealized case of Gaussian turbulence and telescope beam are shown in Figure 1, where we set $\delta=1^{\prime \prime},\left\langle B_{\mathrm{t}}^{2}\right\rangle /\left\langle B_{0}^{2}\right\rangle=0.4$, $\Delta^{\prime}=4^{\prime \prime}, \quad W_{1}=0 . \prime 5, \quad a_{2}=2 \times 10^{-3} \operatorname{arcsec}^{-2}, \quad$ and $a_{4}=-5 \times 10^{-6} \operatorname{arcsec}^{-4}$. The different panels show how the turbulence and ordered autocorrelation functions (in (a)) combine as in Equation (5) to yield the dispersion function (in (b)) obtained from a given data set. The dispersion function is the starting point for the analysis, i.e., the curves shown in (a) are not known a priori. The different length scales between these two functions allow for the separation and recovery of the turbulence autocorrelation function $b^{2}(\ell)$ (solid curve in $(\mathrm{d})$ ) from the ordered component. The contribution of the turbulence correlation length to the broadening of $b^{2}(\ell)$ is apparent from its excess width in comparison to that of the (autocorrelated) telescope beam (broken curve in (d)).

\subsection{Isotropic Gaussian Turbulence Model-Interferometry}

Equation (10) clearly shows the filtering effect of the telescope beam on the power spectrum. Of course this effect also manifests itself on the profile of the corresponding autocorrelation function. For the single-dish case shown in Figure 1, where the filtering is confined to the high-frequency end of the power spectrum, the width of the turbulence autocorrelation function $b^{2}(\ell)$ in panel (d) has a significant contribution stemming from the size of the telescope beam. However, we should not expect the appearance of $b^{2}(\ell)$ or the dispersion function to be exactly the same if the measurement was made with an interferometer since in this case the lowfrequency end of the spectrum will also be strongly filtered. Similarly, neither should we expect the analytical solution to the Gaussian turbulence dispersion problem for interferometry to be given by Equation (13), which was obtained for the single-dish case.

Panel (a) of Figure 2 shows the turbulent power spectrum $\mathcal{R}_{\mathrm{t}}(k) /\left\langle\bar{B}^{2}\right\rangle$ that would be observed with a pencil single-dish beam (i.e., with $W_{1} \rightarrow 0$; solid curve, using the scale on the left) for the example shown in Figure 1 as well as the filter corresponding to the single-dish beam used for these calculations (black broken curve; right scale). To better display the difference in the spectral filtering effect we also show an idealized interferometer beam spectral profile where the lowfrequency component of the single-dish beam profile was removed by subtracting a Gaussian beam of width $W_{2}=2^{\prime \prime}$ (turquoise broken-dotted curve, right scale), to get a better picture of the effect the so-called dirty beam has on the spectrum. More precisely, the spatial profile of the interferometer beam was modeled with

$$
H(\boldsymbol{r})=\frac{1}{2 \pi W_{1}^{2}} e^{-r^{2} / 2 W_{1}^{2}}-\frac{1}{2 \pi W_{2}^{2}} e^{-r^{2} / 2 W_{2}^{2}},
$$

with $W_{1}=0$ ". 5 , as previously stated, and $W_{2}=2^{\prime \prime}$. We again note that $W_{1}$ and $W_{2}$ are for the standard deviation equivalent of the corresponding Gaussian beams. This twin Gaussian profile representation of an interferometer beam is the one we will use for the rest of the analyses presented in this paper. It is, in a sense, an extension of the usual single Gaussian profile commonly used for synthesized beams in interferometry, but it has the advantage of more accurately modeling the spatial filtering of extended structures caused by the beam.

Using this twin Gaussian model for the interferometer beam, it becomes straightforward to generalize the analytical singledish solution for Gaussian turbulence given by Equations (13) and (15) by substituting Equation (18) in the place of Equation (12) into (9). Panel (b) of Figure 2 shows the resulting integrated power spectrum $b^{2}(k)$ for each beam, calculated using Equation (10). The difference in filtering between the two kinds of measurements is made clear.

The interferometer solution to the idealized Gaussian turbulence problem is given by

$$
\begin{aligned}
1-\langle\cos [\Delta \Phi(\ell)]\rangle= & \sum_{j=1}^{\infty} a_{2 j} \ell^{2 j}+\left[\frac{N}{1+N\left\langle B_{0}^{2}\right\rangle /\left\langle B_{\mathrm{t}}^{2}\right\rangle}\right] \\
& \times\left\{\frac{1}{N_{1}}\left[1-e^{-\ell^{2} / 2\left(\delta^{2}+2 W_{1}^{2}\right)}\right]\right. \\
& \frac{1}{N_{2}}\left[1-e^{-\ell^{2} / 2\left(\delta^{2}+2 W_{2}^{2}\right)}\right] \\
& \left.-\frac{2}{N_{12}}\left[1-e^{-\ell^{2} / 2\left(\delta^{2}+W_{1}^{2}+W_{2}^{2}\right)}\right]\right\},
\end{aligned}
$$

with $N_{1}$ still given by Equation (14) and

$$
\begin{gathered}
N_{2}=\frac{\left(\delta^{2}+2 W_{2}^{2}\right) \Delta^{\prime}}{\sqrt{2 \pi} \delta^{3}} \\
N_{12}=\frac{\left(\delta^{2}+W_{1}^{2}+W_{2}^{2}\right) \Delta^{\prime}}{\sqrt{2 \pi} \delta^{3}} \\
N=\left(\frac{1}{N_{1}}+\frac{1}{N_{2}}-\frac{2}{N_{12}}\right)^{-1} .
\end{gathered}
$$

The corresponding normalized signal-integrated turbulence autocorrelation function is

$$
\begin{aligned}
b^{2}(\ell)= & {\left[\frac{N}{1+N\left\langle B_{0}^{2}\right\rangle /\left\langle B_{\mathrm{t}}^{2}\right\rangle}\right]\left[\frac{1}{N_{1}} e^{-\ell^{2} / 2\left(\delta^{2}+2 W_{1}^{2}\right)}\right.} \\
& \left.+\frac{1}{N_{2}} e^{-\ell^{2} / 2\left(\delta^{2}+2 W_{2}^{2}\right)}-\frac{2}{N_{12}} e^{-\ell^{2} / 2\left(\delta^{2}+W_{1}^{2}+W_{2}^{2}\right)}\right] .
\end{aligned}
$$



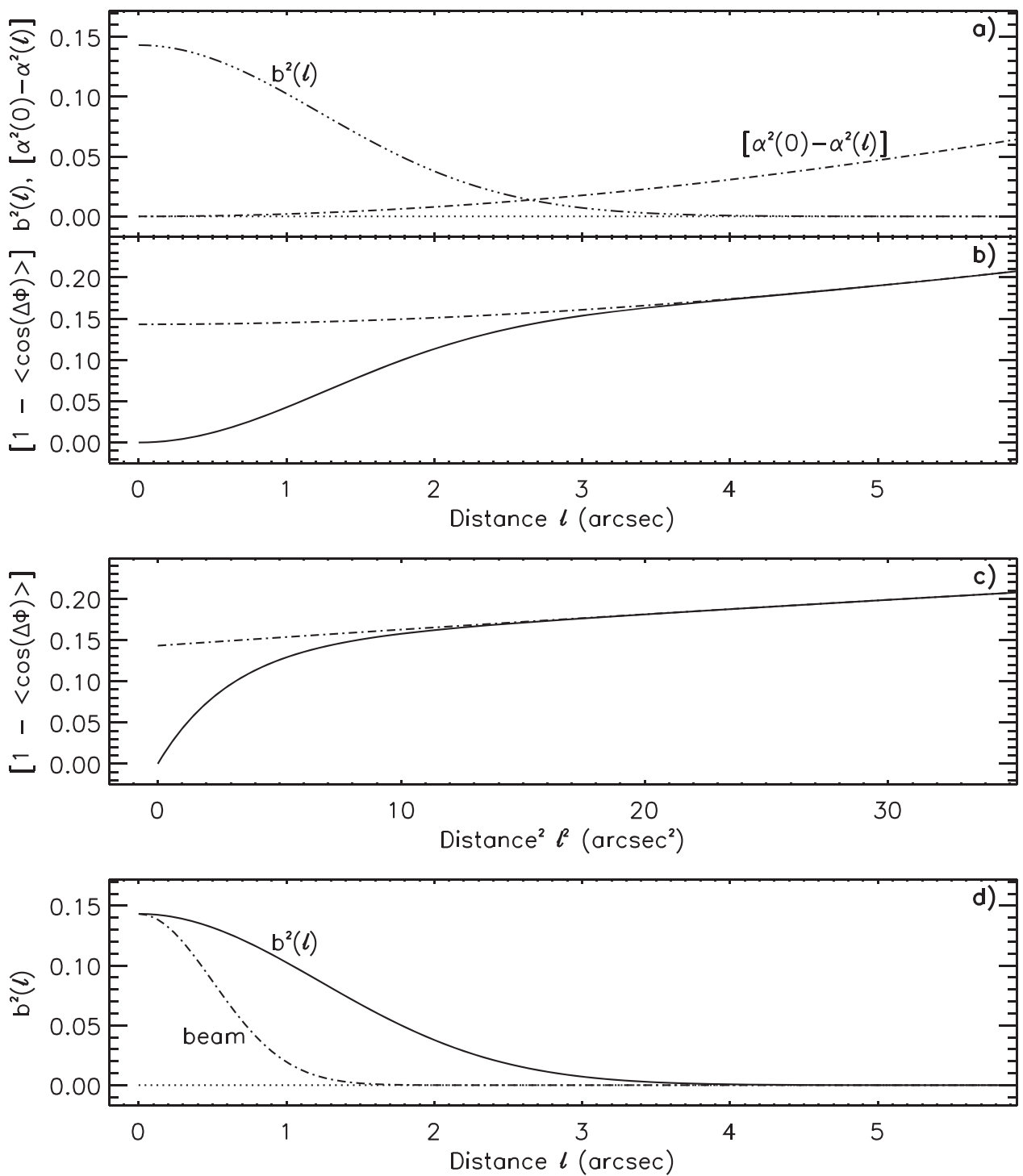

Figure 1. Example of an idealized case of Gaussian turbulence and telescope beam, where we set the turbulence correlation length $\delta=1^{\prime \prime}$, the turbulent-toordered magnetic energy ratio $\left\langle B_{\mathrm{t}}^{2}\right\rangle /\left\langle B_{0}^{2}\right\rangle=0.4$, the effective depth $\Delta^{\prime}=4^{\prime \prime}$, the telescope beam width $W_{1}=0.0^{\prime \prime} 5$, and the large-scale coefficients $a_{2}=2 \times 10^{-3} \operatorname{arcsec}^{-2}$ and $a_{4}=-5 \times 10^{-6} \operatorname{arcsec}^{-4}$. (a) The turbulence $\left(b^{2}(\ell)\right.$; Equation (15)) and the ordered $\left(\alpha^{2}(0)-\alpha^{2}(\ell)\right)$ autocorrelation functions, plotted against $\ell$, combine as in Equation (5) to yield the dispersion function in (b). The solid and broken-dotted curves in (b) are for the dispersion function and $b^{2}(0)+\left[\alpha^{2}(0)-\alpha^{2}(\ell)\right]$, respectively. For a given data set, the dispersion function is the starting point for the analysis, i.e., the curves shown in (a) are not known a priori. (c) Same as (b) but plotted as a function of $\ell^{2}$ to better show the difference in their length scales. (d) The different length scales allow for the separation of the turbulence autocorrelation function $b^{2}(\ell)$ (solid curve) from $b^{2}(0)+\left[\alpha^{2}(0)-\alpha^{2}(\ell)\right]$ in $(\mathrm{b})$ and its recovery. The contribution of the turbulence correlation length to the broadening of $b^{2}(\ell)$ is apparent from its excess width in comparison to that of the (autocorrelated) telescope beam (brokendotted curve, normalized for convenience).

For a given polarimetry map, estimates for $\delta,\left\langle B_{\mathrm{t}}^{2}\right\rangle /\left\langle B_{0}^{2}\right\rangle$, and $a_{2 j}$ are obtained by fitting the right-hand side of Equation (19) to the data (on the left-hand side). It is also straightforward to verify that this interferometry solution tends to the single-dish solution when $W_{2} \rightarrow \infty$, as would be expected. Figure 3 shows the corresponding dispersion function resulting from the interferometer case of the Gaussian turbulence example used in Figure 1. We have kept the ordered component the same to facilitate the comparison between the single-dish and interferometer cases. We note the difference in the appearance of the large-scale component in relation to the dispersion function in panels (b) and (c), which brings an oscillatory behavior in the autocorrelation function $b^{2}(\ell)$ in panel (d). These oscillations ensure that

$$
\begin{aligned}
2 \pi \int_{0}^{\infty} b^{2}(\ell) \ell d \ell & =b^{2}\left(\boldsymbol{k}_{v}=0\right) \\
& =0,
\end{aligned}
$$

as required for such an interferometer beam. We finally note that the turbulent-to-total energy ratio is still given by Equation (16), but with $N_{1}$ replaced by $N$.

\section{RESULTS-ANALYSIS OF CARMA DATA}

We now apply our Gaussian turbulence analysis to interferometry data on the W3(OH), W3 Main, and DR21 $(\mathrm{OH})$ molecular clouds obtained with CARMA at a frequency 


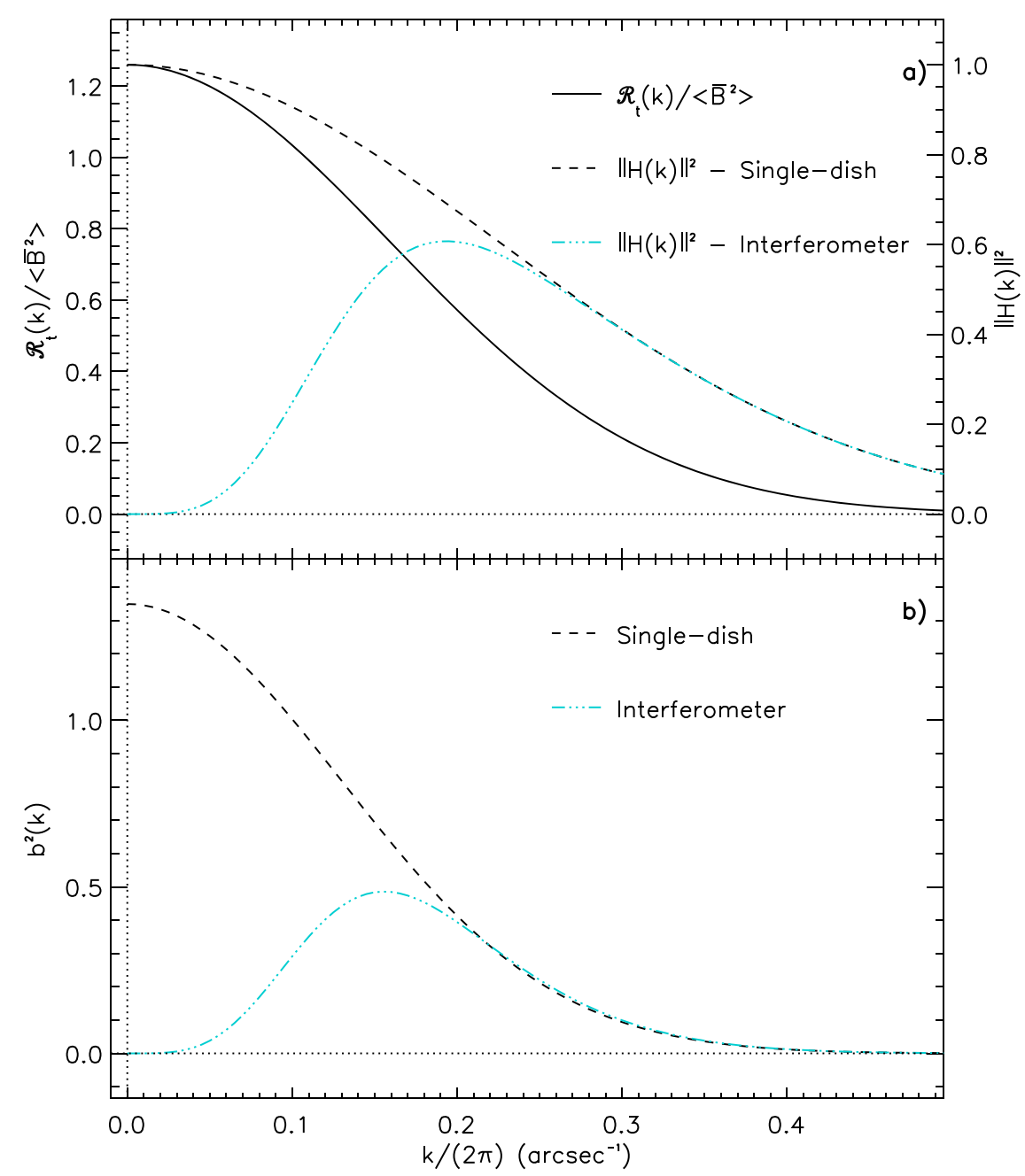

Figure 2. Top: turbulent power spectrum $\mathcal{R}_{\mathrm{t}}(k) /\left\langle\bar{B}^{2}\right\rangle$ that would be observed with a pencil single-dish beam (i.e., with $W_{1} \rightarrow 0$; black solid curve, using the scale on the left), and the filters corresponding to the single-dish beam for the example of Figure 1 (black broken curve) and a corresponding idealized interferometer beam with $W_{2}=2^{\prime \prime}$ (turquoise broken-dotted curve; both beam profiles use the scale on the right). Bottom: the turbulent power spectra $b^{2}(k)$ that would be obtained with the single-dish (black broken curve) and the interferometer (turquoise broken-dotted curve) beams.

of $223.821 \mathrm{GHz}$. These data were presented and discussed in detail in Hull et al. (2014), where more information will be found. In all cases, we used data points where $p \geqslant 2 \sigma_{p}$, with $p$ and $\sigma_{p}$ the polarization level and its uncertainty, respectively, and $I \geqslant 3 \sigma_{I}$, with $I$ and $\sigma_{I}$ the Stokes $I$ intensity and its uncertainty, respectively. The geometric mean of the full width at half magnitude (FWHM) of the synthesized beam (divided by $\sqrt{8 \ln (2)}$ ) was used for $W_{1}$, while the value for $W_{2}$ was chosen to account for the low-frequency response and filtering of the telescope dirty beam (see below). The value $\Delta^{\prime}$ was determined by calculating the (half)width of the autocorrelation function of the polarized flux at half of the maximum amplitude. The dispersion functions were then calculated from the polarization data using the left-hand side of Equation (19) and the Gaussian model fitted for $\delta,\left\langle B_{\mathrm{t}}{ }^{2}\right\rangle /\left\langle B_{0}^{2}\right\rangle$, and $a_{2 j}$ on the right-hand side of that same equation. Although our aforementioned selection criteria for $\sigma_{p}$ and $\sigma_{I}$ reduce the impact of measurement uncertainties on the analysis, we note that the dispersion function is corrected for corresponding biases (not unlike the way the polarization level is usually corrected in linear polarization data). More details for these calculations and the propagation of errors will be found in the Appendix.

To provide an estimate of the magnetic field strength for each source, we measured the total flux $S_{\nu}$ on the corresponding map and converted it to a total mass

$$
M_{\mathrm{gas}}=\frac{S_{\nu} d^{2}}{\kappa_{\nu} B_{\nu}\left(T_{\mathrm{d}}\right)}
$$

where $d$ is the distance to the source, $T_{\mathrm{d}}$ is the dust temperature, and $\kappa_{\nu}$ and $B_{\nu}\left(T_{\mathrm{d}}\right)$ are, respectively, the enhanced mass absorption cross section and the Planck function at the frequency of the observations (Chini et al. 1997). We then estimated the approximate size of the source on the sky to determine its volume and mean mass density, the latter being converted to a number density by assuming a mean molecular mass of 2.3. The mean mass density estimate $\rho$ was then used with suitable line width information for the one-dimensional turbulence velocity dispersion $\sigma(v)$, and the value for $\left\langle B_{\mathrm{t}}^{2}\right\rangle /\left\langle B^{2}\right\rangle$ from our dispersion analysis to evaluate the strength of the plane of the sky component of the magnetic field with the DCF method (Davis 1951; Chandrasekhar \& Fermi 1953)

$$
B_{0} \simeq \sqrt{4 \pi \rho} \sigma(v)\left[\frac{\left\langle B_{\mathrm{t}}^{2}\right\rangle}{\left\langle B^{2}\right\rangle}\right]^{-1 / 2} .
$$



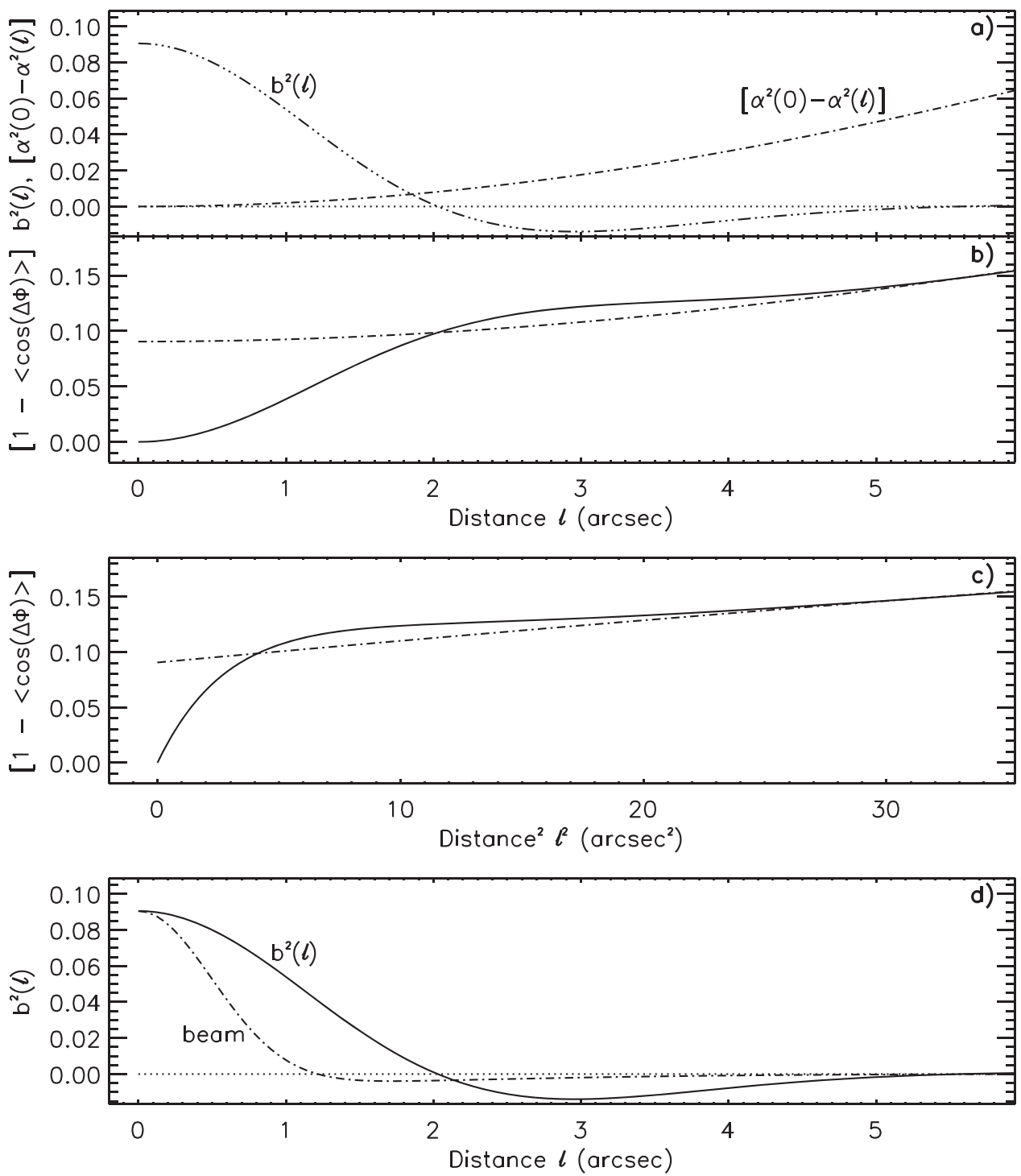

Figure 3. Same as Figure 1 but for an interferometer with $W_{2}=2^{\prime \prime}$. The large-scale component is once again shown with the broken-dotted curve in panels (b) and (c).

Table 2

Dispersion Analysis Results

\begin{tabular}{lcccccccc}
\hline \hline Source & $S_{\nu}(\mathrm{Jy})^{\mathrm{a}}$ & $M_{\mathrm{gas}}\left(M_{\odot}\right)$ & $\rho\left(\mathrm{g} \mathrm{cm}^{-3}\right)$ & $\sigma(v)\left(\mathrm{km} \mathrm{s}^{-1}\right)$ & $\delta(\mathrm{mpc})$ & $\left\langle B_{\mathrm{t}}^{2}\right\rangle /\left\langle B^{2}\right\rangle$ & $N^{\mathrm{b}}$ & $B_{0}(\mathrm{mG})^{\mathrm{c}}$ \\
\hline W3(OH) & 5.7 & 149 & $5.0 \times 10^{-18}$ & 1.1 & $19.0 \pm 0.2$ & $0.58 \pm 0.01$ & $4.67 \pm 0.04$ & 1.1 \\
W3 Main & 3.5 & 83 & $1.8 \times 10^{-18}$ & 1.2 & $22.2 \pm 0.3$ & $0.74 \pm 0.01$ & $9.58 \pm 0.04$ & 0.7 \\
DR21(OH) & 4.6 & 65 & $7.8 \times 10^{-18}$ & 1.0 & $12.3 \pm 0.2$ & $0.70 \pm 0.01$ & $6.91 \pm 0.07$ & 1.2 \\
\hline
\end{tabular}

Notes.

${ }^{\mathrm{a}}$ From Equation (25) with $T_{\mathrm{d}}=30 \mathrm{~K}$ and $\kappa_{\nu}=0.02 \mathrm{~cm}^{2} \mathrm{~g}^{-1}$ for all sources.

${ }^{\mathrm{b}}$ Number of turbulent cells in the column of gas subtended by the telescope beam.

${ }^{c}$ Accurate within a factor of approximately three.

Finally, although there are other suitable candidates for a dispersion analysis in the TADPOL sample of Hull et al. (2014), W3 $(\mathrm{OH})$, W3 Main, and DR21(OH) were chosen because of the large number of independent polarization measurements available for these sources (resulting in better statistics) and their relative closeness. We present the results for each sources below and provide a summary in Table 2 .

\section{1. $\mathrm{W} 3(\mathrm{OH})$}

$\mathrm{W} 3(\mathrm{OH})$ is an active high-mass star-forming region located some $2040 \mathrm{pc}$ away at R.A.(J2000) $=2^{\mathrm{h}} 27^{\mathrm{m}} 03^{\mathrm{s}}$.9, decl. $(\mathrm{J} 2000)=-61^{\circ} 52^{\prime} 24^{\prime \prime} 6$ (Hachisuka et al. 2006). The polarization map on which our analysis was performed can be found in Figure 5 of Hull et al. (2014). The FWHM of the synthesized telescope beam for these observations is 

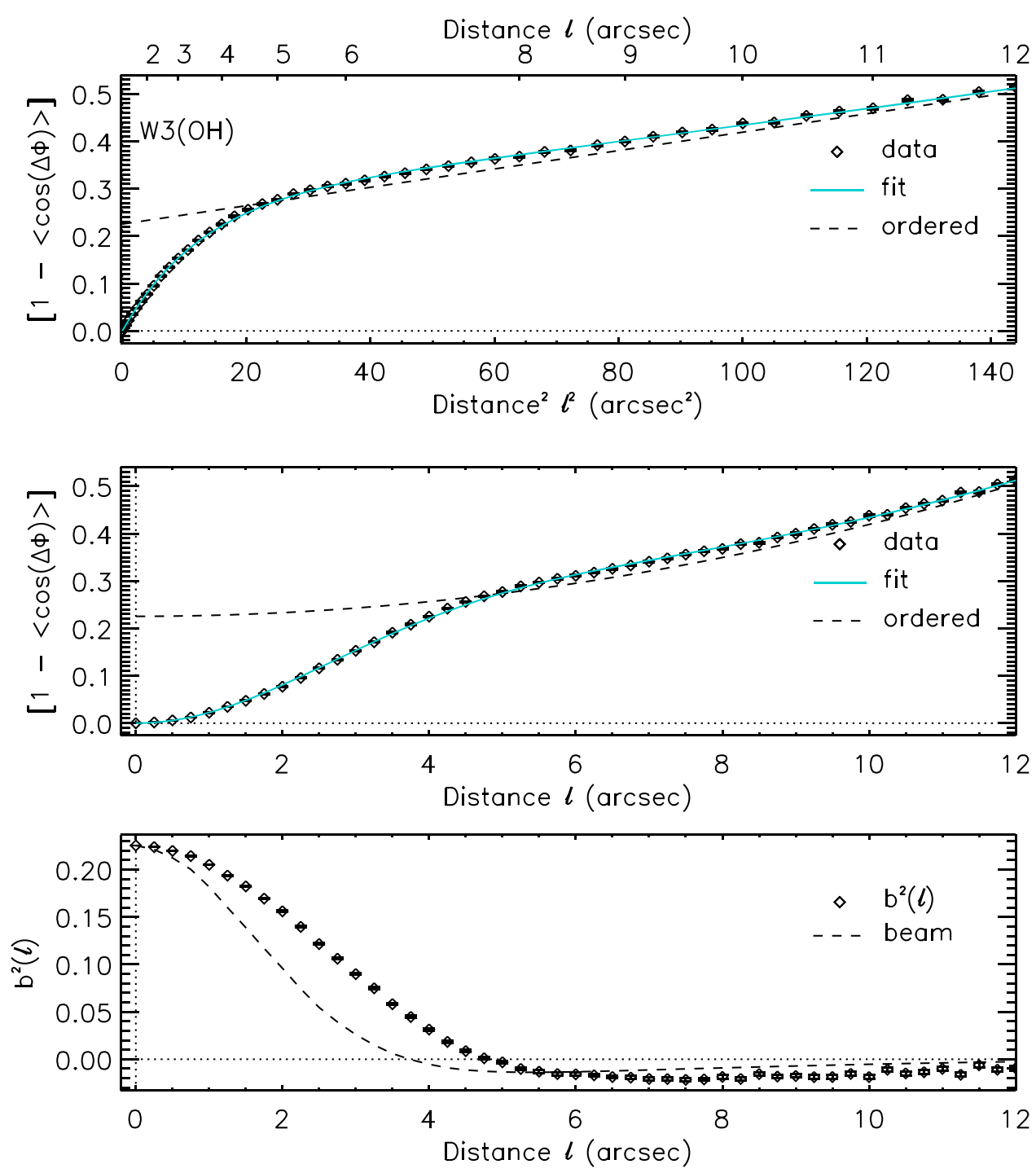

Figure 4. Dispersion analysis of the CARMA W3(OH) data. Top: the dispersion function $1-\langle\cos [\Delta \Phi(\ell)]\rangle$ of the data (symbols) plotted as a function of $\ell^{2}$ to better show the difference in scale for the turbulent and ordered components. The ordered component (see Equation (8)) is also shown with the broken curve. The leastsquares fit of the Gaussian turbulence model given in Equation (19) is plotted in turquoise (solid curve). Middle: same as the top panel, but as a function of $\ell$. Bottom: the resulting signal-integrated turbulence autocorrelation function $b^{2}(\ell)$ (symbols) is seen to exhibit an excess in its width relative to that of the autocorrelated beam (broken curve; the beam function is given by Equation (18)). The fit to the data yields a turbulence correlation length $\delta \simeq 1$ ". $92 \pm 0$ "' 02 (or $19.0 \pm 0.2 \mathrm{mpc}$ at the distance of $\mathrm{W} 3(\mathrm{OH})$ ) and a turbulent-to-total magnetic energy ratio $\left\langle B_{\mathrm{t}}^{2}\right\rangle /\left\langle B^{2}\right\rangle \simeq 0.58 \pm 0.01$.

2 !. $8 \times 2$ ". 6 at a PA $=12^{\circ} .4$. The beam sizes (i.e., their standard deviation equivalent) used for the twin Gaussian profile are $W_{1}=1$." 2 and $W_{2}=6$." 6 , while $\Delta^{\prime}=10$." 5 as determined from the autocorrelation function of the polarized flux. The results of the dispersion analysis for this source are shown in Figure 4, where the top panel is a plot of the dispersion function $1-\langle\cos [\Delta \Phi(\ell)]\rangle$ of the data (symbols) as a function of $\ell^{2}$ to better show the difference in scale between the turbulent and ordered components. The center panel of the figure reveals the same information, but this time with the dispersion function plotted as a function of $\ell$. The bottom panel yields the resulting signal-integrated turbulence autocorrelation function $b^{2}(\ell)$ (symbols), which is seen to exhibit an excess in its width relative to that of the autocorrelated beam (broken curve; the beam function is given by Equation (18)); this is a signature of the intrinsic magnetized turbulence present in the medium under study. The fit to the data yields a magnetized turbulence correlation length $\delta=1$.' $92 \pm 0$. ". 02 (or $19.0 \pm 0.2 \mathrm{mpc}$ at the distance of $\mathrm{W} 3(\mathrm{OH})$ ) and a turbulent-to-total magnetic energy ratio $\left\langle B_{\mathrm{t}}^{2}\right\rangle /\left\langle B^{2}\right\rangle=0.58 \pm 0.01$. It is therefore apparent that a significant fraction of the magnetic energy is in the form of turbulence. The number of turbulent cells $N$ contained in the column of gas subtended by the telescope beam was found to be $N=4.67 \pm 0.04$. Although the fit to the data is good, it is important to note that the values obtained for $\delta,\left\langle B_{\mathrm{t}}^{2}\right\rangle /\left\langle B^{2}\right\rangle$, and $N$ are only valid within the framework of the Gaussian turbulence model. This is an idealization that is certainly not realized for molecular clouds and the ISM in general, i.e., turbulence is not Gaussian in nature. Furthermore, we do not precisely know the value of the effective depth of clouds $\Delta^{\prime}$, and this can have a significant effect on the uncertainties derived in the analysis. For example, the estimated values for the turbulent-to-ordered magnetic energy ratio $\left\langle B_{\mathrm{t}}^{2}\right\rangle /\left\langle B_{0}^{2}\right\rangle$ and the number of turbulent cells $N$, and their uncertainties, scale linearly with $\Delta^{\prime}$.

Figure 5 shows the signal-integrated turbulence power spectrum $b^{2}(k) \quad\left(k=\left|\boldsymbol{k}_{v}\right|\right.$; symbols $)$ obtained through the 


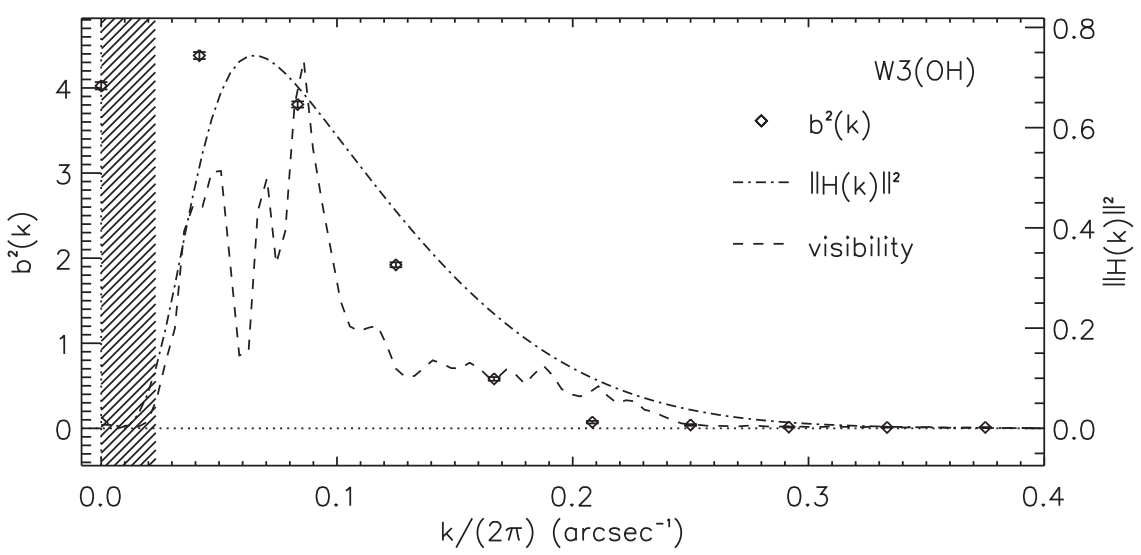

Figure 5. Signal-integrated turbulence power spectrum $b^{2}(k)$ (symbols) obtained through the Fourier transform of $b^{2}(\ell)$ in the bottom panel of Figure 4 for W3(OH). The spectral shape of the autocorrelated dirty beam is also shown (broken curve; "visibility") to better visualize the spectral filtering imposed on the data by the interferometer. The synthesized beam for the data has $W_{1}=1$ "' 2 , while we subtracted another Gaussian beam component with $W_{2}=66^{\prime \prime} 6$ to model the low-frequency filtering. The resulting autocorrelated twin Gaussian beam has the spectral shape shown by the broken-dotted curve $\left(\|H(k)\|^{2}\right)$. The shaded part of the spectrum at low frequencies outlines the approximate region where data cannot be trusted in view of the filtering due to the telescope dirty beam. For this analysis, only the datum at $k=0$ is affected (see text).

Fourier transform of $b^{2}(\ell)$, taken from the bottom panel of Figure 4. The spectral shape of the autocorrelated dirty beam is also shown (broken curve; "visibility") to better visualize the spectral filtering imposed on the data by the interferometer. According to our earlier statement, the synthesized beam for the data has $W_{1}=1$ !' 2 , while we subtracted another Gaussian beam component with $W_{2}=6$." 6 to model the low-frequency filtering. The resulting autocorrelated twin Gaussian beam has the spectral shape shown by the broken-dotted curve $\left(\|H(k)\|^{2}\right)$ and corresponds to the Fourier transform of the autocorrelated beam shown in the bottom panel of Figure 4. We note that the power spectrum $b^{2}(k)$ does not go to zero at $k=0$, as would be expected from Equation (24). This is likely due to the fact that the dispersion function cannot be evaluated at sufficiently large enough values for $\ell$ (i.e., it is truncated), which causes aliasing in the power spectrum near $k=0$, although it is also possible that the CLEANing process of the interferometry data could add signals at low frequencies in the power spectrum (Thompson et al. 2004). Whatever the case, it follows that the value of $b^{2}(k=0)$ is erroneous and should not be trusted. The same can be said for the shaded part of the spectrum at low frequencies, which outlines the approximate region that is heavily filtered by the telescope dirty beam.

We can provide an approximate value for the magnetic field strength (plane-of-the-sky component) from the fit parameters using the DCF method (Davis 1951; Chandrasekhar \& Fermi 1953) with Equation (26). The total flux measured from the CARMA map is $S_{\nu}=5.7 \mathrm{Jy}$, which from Equation (25) translates to a total mass of $M_{\text {gas }}=149 M_{\odot}$ for this object with $T_{\mathrm{d}}=30 \mathrm{~K}$ and $\kappa_{\nu} \simeq 0.02 \mathrm{~cm}^{2} \mathrm{~g}^{-1}$ (Chini et al. 1997). This mass is approximately contained within an ellipse of $\simeq 10^{\prime \prime} \times 6^{\prime \prime}$ in size (FWHM), yielding a mean density of $\simeq 1.3 \times 10^{6} \mathrm{~cm}^{-3}$ (or $\rho \simeq 5.0 \times 10^{-18} \mathrm{~g} \mathrm{~cm}^{-3}$ ). For a measure of $\sigma(v)$ we follow the prescription given in Paper II and use the line width from a suitable ion of similar effective density as that at which the dust emission is detected. We thus find the line width of $2.8 \mathrm{~km} \mathrm{~s}^{-1}$ from our own $\mathrm{HCO}^{+}(4 \rightarrow 3)$ (unpublished) measurements obtained at the Caltech Submillimeter Observatory (CSO) at the peak flux position in $\mathrm{W} 3(\mathrm{OH})$. When scaling down this value to account for the larger CSO beam (FWHM of $\simeq 18^{\prime \prime}$ ) using a $\sim L^{0.5}$ scaling law for the velocity dispersion (where $L$ is the length-scale), we have $\sigma(v) \sim 1.1 \mathrm{~km} \mathrm{~s}^{-1}$, and we obtain $B_{0} \sim 1.1 \mathrm{mG}$ from Equation (26). Errors in $B_{0}$ are very difficult to quantify. Since it is subject to significant uncertainties in $\Delta^{\prime}, \rho$, and $\sigma(v)$, as well as those intrinsic to the DCF method, we suggest that our estimate for $B_{0}$ determined this way is accurate to a factor of about three. Nonetheless, this value is consistent with the Zeeman $\mathrm{CN}(1 \rightarrow 0)$ measurement of Falgarone et al. (2008) who also found a value of $\sim 1.1 \mathrm{mG}$ for the line of sight component of the magnetic field in this source.

\subsection{W3 Main}

W3 Main is a massive star-forming region located some $1950 \mathrm{pc}$ away in the outer region of the Galaxy. The CARMA map on which our analysis is performed is centered on the position of W3 IRS5 at R.A.(J2000) $=2^{\mathrm{h}} 25^{\mathrm{m}} 40 \mathrm{~s} 6$, decl. $(\mathrm{J} 2000)=-62^{\circ} 05^{\prime} 51^{\prime \prime} 6$, and will be found in Figure 4 of Hull et al. (2014). The FWHM of the synthesized telescope beam for these observations is 3 ." $0 \times 22^{\prime \prime} 9$ at a PA $=-27^{\circ} .0$, and the beam sizes used for the twin Gaussian profile in our analysis are $W_{1}=11^{\prime \prime} 2$ and $W_{2}=9$ !' 3 , while $\Delta^{\prime}=31{ }^{\prime \prime} 3$. The results of the dispersion analysis for this source are presented in Figures 6 and 7. As for W3(OH), our model yielded a good fit to the data with $\delta=2$ !" $35 \pm 0$ " 03 (or $22.2 \pm 0.3 \mathrm{mpc}$ at the distance of W3 Main), $\left\langle B_{\mathrm{t}}^{2}\right\rangle /\left\langle B^{2}\right\rangle=0.74 \pm 0.01$, and $N=9.58 \pm 0.04$.

The total flux measured from the CARMA map is $3.5 \mathrm{Jy}$, yielding a total mass of $83 M_{\odot}$ (we again use a dust temperature of $30 \mathrm{~K}$ and $\kappa_{\nu} \simeq 0.02 \mathrm{~cm}^{2} \mathrm{~g}^{-1}$ ) approximately contained within two circles of 9!" 8 and 15"'6 (FWHM), respectively, as seen on the plane of the sky, which imply a mean density of $\simeq 4.7 \times 10^{5} \mathrm{~cm}^{-3} \quad$ (or $\rho \simeq 1.8 \times 10^{-18} \mathrm{~g} \mathrm{~cm}^{-3}$ ). Using a velocity dispersion of $3.0 \mathrm{~km} \mathrm{~s}^{-1}$ from the CSO $\mathrm{HCO}^{+}(4 \rightarrow 3$ ) observations of Houde et al. (2000b), employing the same power law scaling as before, we find $\sigma(v) \sim 1.2 \mathrm{~km} \mathrm{~s}^{-1}$ and $B_{0} \sim 0.7 \mathrm{mG}$. Presumably precise within a factor of three, we find a magnetic field strength close to that calculated for $\mathrm{W} 3(\mathrm{OH})$. 

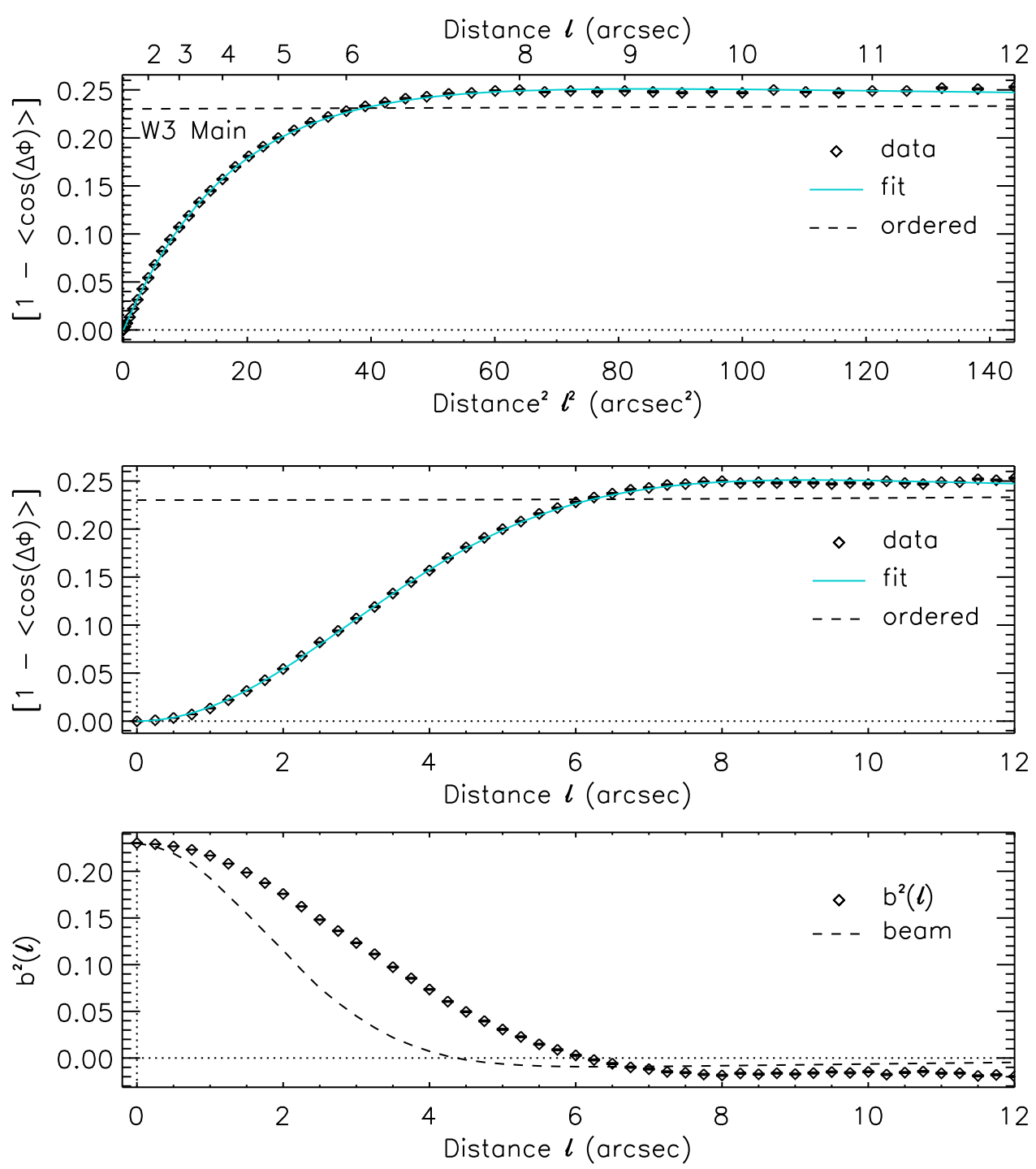

Figure 6. Same as Figure 4 but for W3 Main, where the fit to the data yields $\delta \simeq 2$ ". $35 \pm 0$ " 03 (or $22.2 \pm 0.3$ mpc at the distance of W3 Main) and $\left\langle B_{\mathrm{t}}^{2}\right\rangle /\left\langle B^{2}\right\rangle \simeq 0.74 \pm 0.01$.

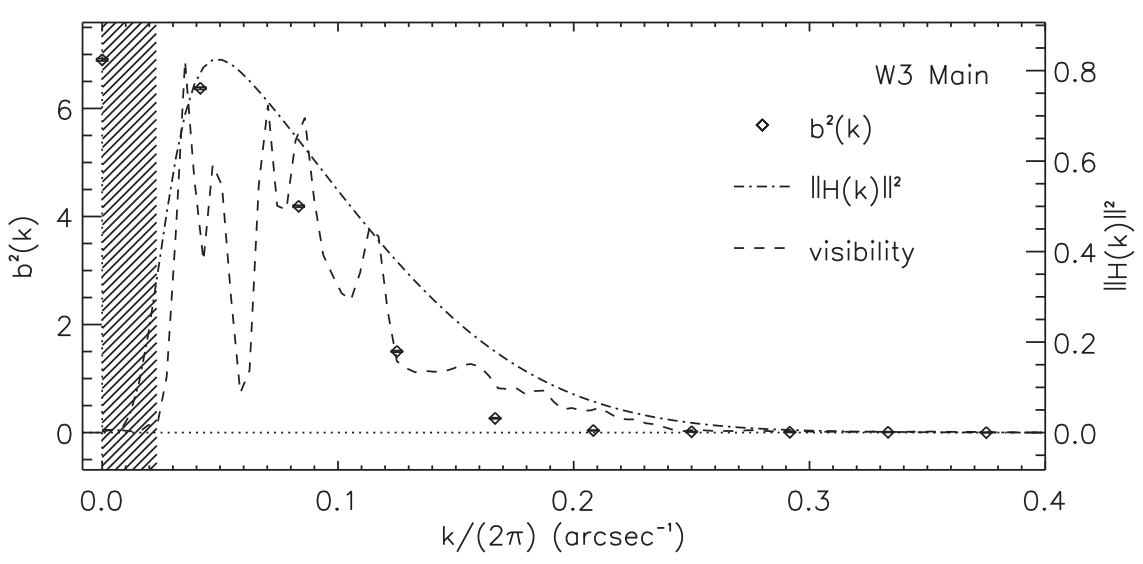

Figure 7. Same as Figure 5 but for W3 Main, where $W_{1}=1$ !" 2 and $W_{2}=9$ !" 3 .

\section{3. $\mathrm{DR} 21(\mathrm{OH})$}

$\mathrm{DR} 21(\mathrm{OH})$ is a massive star-forming region located some $1500 \mathrm{pc}$ away. The CARMA map on which our analysis is performed is centered at R.A. $(\mathrm{J} 2000)=20^{\mathrm{h}} 39^{\mathrm{m}} 01^{\mathrm{s}} .1$, decl. $(\mathrm{J} 2000)=42^{\circ} 22^{\prime} 29^{\prime \prime}$. 0 , and will be found in Figure 32 of Hull et al. (2014). The FWHM of the synthesized telescope beam for these observations is 2 !" $7 \times 2$ !" 6 at a $\mathrm{PA}=37$ !" 5 , yielding $W_{1}=1$ !" 1 and $W_{2}=7$ !" 6 for our analysis, while $\Delta^{\prime}=13$ ". 5 . The results of the dispersion analysis for this 

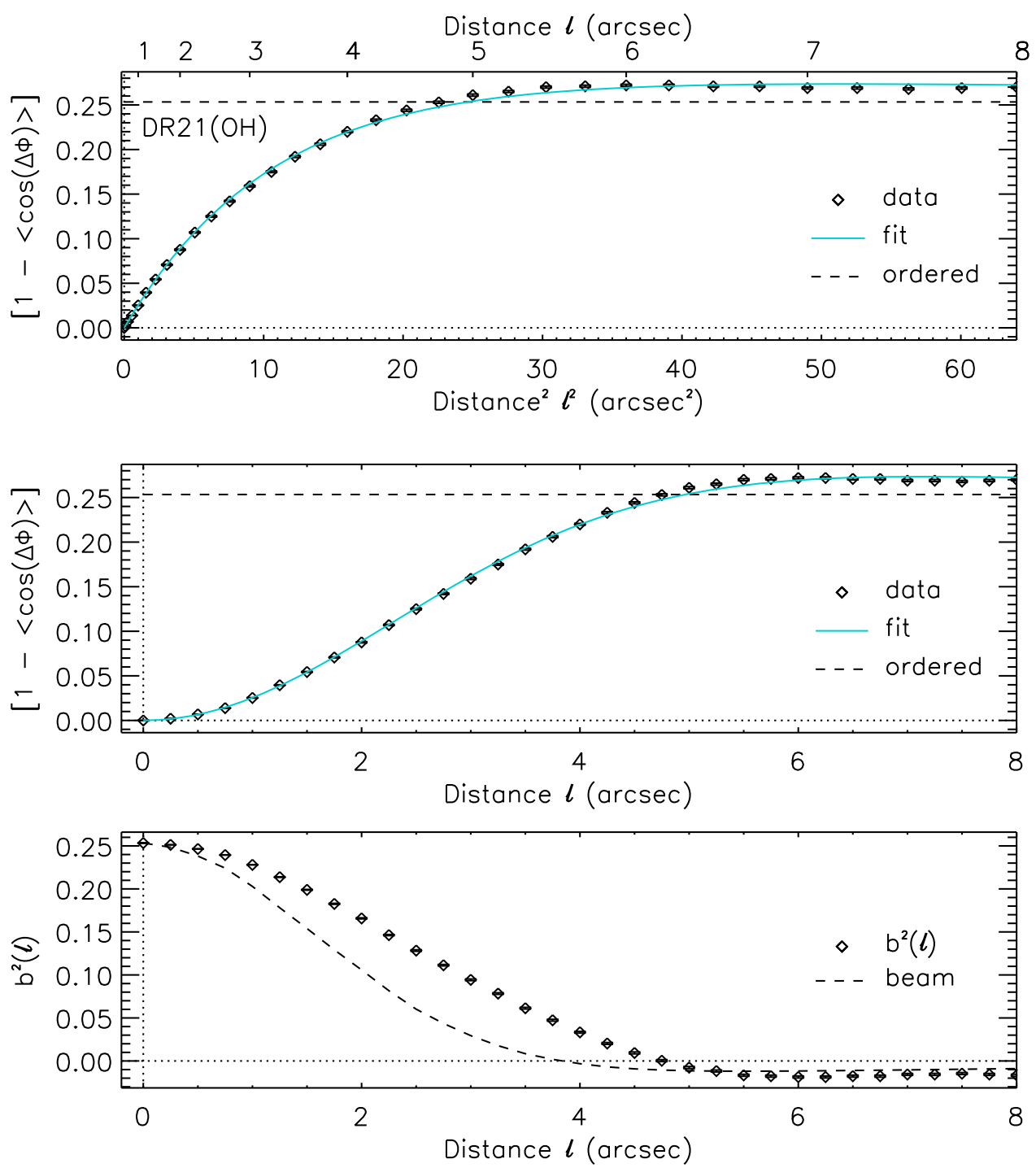

Figure 8. Same as Figure 4 but for DR21(OH), where the fit to the data yields $\delta \simeq 1$.' $69 \pm 00^{\prime \prime} 02$ (or $12.3 \pm 0.2 \mathrm{mpc}$ at the distance of W3 Main) and $\left\langle B_{\mathrm{t}}^{2}\right\rangle /\left\langle B^{2}\right\rangle \simeq 0.70 \pm 0.01$.

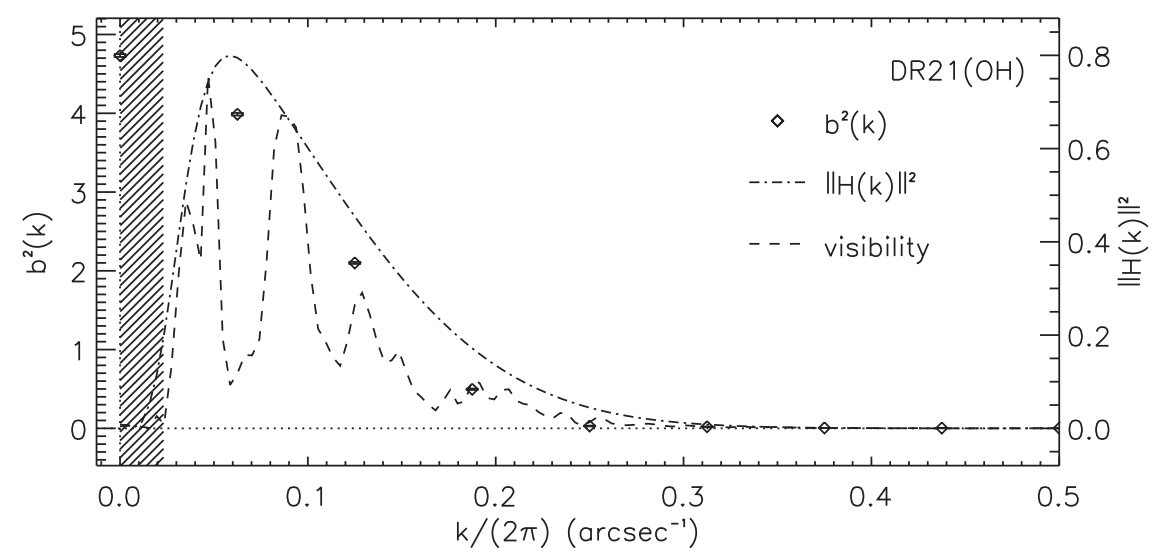

Figure 9. Same as Figure 5 but for DR21(OH), where $W_{1}=1$ ". 1 and $W_{2}=7$ " 6 .

source are presented in Figures 8 and 9. As for the other sources studied here, our model yielded a good fit to the data providing $\delta=1 . \prime 69 \pm 0$ !' 02 (or $12.3 \pm 0.2 \mathrm{mpc}$ at the distance of $\operatorname{DR} 21(\mathrm{OH})),\left\langle B_{\mathrm{t}}^{2}\right\rangle /\left\langle B^{2}\right\rangle=0.70 \pm 0.01$, and $N=6.91 \pm 0.07$.
The total flux measured from the CARMA map is $4.6 \mathrm{Jy}$, yielding a total mass of $65 M_{\odot}$ (we again use a dust temperature of $30 \mathrm{~K}$ and $\kappa_{\nu} \simeq 0.02 \mathrm{~cm}^{2} \mathrm{~g}^{-1}$ ). This result is about a factor of two smaller than the $150 M_{\odot}$ estimate obtained by Girart et al. (2013) with previous SMA interferometry data at $880 \mu \mathrm{m}$. We 
find that this mass is approximately contained within a circle of 12 ". 4 (FWHM), as seen on the plane of the sky, giving a mean density of $\simeq 2.0 \times 10^{6} \mathrm{~cm}^{-3}$ (or $\rho \simeq 7.8 \times 10^{-18} \mathrm{~g} \mathrm{~cm}^{-3}$ ). We use the velocity dispersion of $1 \mathrm{~km} \mathrm{~s}^{-1}$ from the $\mathrm{H}^{13} \mathrm{CO}^{+}(4 \rightarrow 3)$ SMA observations of Girart et al. (2013) and find $B_{0} \sim 1.2 \mathrm{mG}$. Still precise within a factor of approximately three, this value is consistent with the Zeeman $\mathrm{CN}(1 \rightarrow 0)$ measurement of Falgarone et al. (2008) who found values of 0.36 and $0.71 \mathrm{mG}$ for line of sight component of the magnetic field for the MM1 and MM2 components in this source. The same type of agreement exists with the earlier result of Hezareh et al. (2010, 2014), who obtained $B_{0} \simeq 0.7 \mathrm{mG}$ with single-dish $\mathrm{H}^{13} \mathrm{CN}(4 \rightarrow 3)$ and $\mathrm{H}^{13} \mathrm{CO}^{+}(4 \rightarrow 3)$ data using the ion-neutral line width comparison technique developed by $\mathrm{Li} \&$ Houde (2008; see also Houde et al. 2000a, 2000b, 2001). Furthermore, our value of $\delta=12.3 \mathrm{mpc}$ is in good agreement with the $8.5 \mathrm{mpc}$ dissipation scale they measured using the same method, as we would expect that scale to be shorter than the turbulence correlation length (see Papers II and III). Finally, it is interesting to note that our analysis also gives values that are reasonably close to those obtained by Girart et al. (2013) through their analysis of the aforementioned SMA interferometry data that yielded $B_{0} \simeq 2.1 \mathrm{mG}$ and $\delta \simeq 16.9 \mathrm{mpc}$, although they used the dispersion analysis technique developed for single-dish data in Paper II (see below).

\section{SUMMARY AND CONCLUSION}

Although an idealization that is not likely to be realized in the ISM, the Gaussian turbulence model provides a useful analytical solution to the angular dispersion analysis problem, either for single-dish or interferometry observations, in that it allows the quantification of key parameters characterizing magnetized turbulence in the ISM. It is therefore interesting to note that despite this idealization, our application of the model to interferometry has yielded an excellent fit for each of the three data sets presented in this paper.

As noted earlier in Section 2.2, this new solution for interferometry differs from the one for single-dish data by a dependency on $W_{2}$, the width of the Gaussian function subtracted to the synthesized interferometry beam (of width $W_{1}$ ) to account for the filtering of extended structures (i.e., low spatial frequencies). Since this interferometry solution for the angular dispersion function (i.e., Equation (19)) tends to the single-dish solution (i.e., Equation (13)) in the limit when $W_{1} \ll W_{2}$, it is at this point interesting to assess the errors that would ensue if the single-dish model was used for the analysis of our interferometry data. More precisely, for the three sources, all with good $u v$-coverage at low frequencies, we have $0.1 \lesssim W_{1} / W_{2} \lesssim 0.2$ and find that the errors we incur are relatively modest. That is, the single-dish model overestimates the values for $b^{2}(0)$ and the intrinsic turbulent-to-ordered magnetic energy ratio $\left\langle B_{\mathrm{t}}^{2}\right\rangle /\left\langle B_{0}^{2}\right\rangle$ by approximately $10 \%$ to $20 \%$, while the turbulence correlation length $\delta$ and the number of turbulent cells $N$ (or $N_{1}$ for the single-dish) are underestimated by $\sim 5 \%$. As was earlier alluded to, it is likely that the CLEAN algorithm used in processing the interferometry data could inject some signals at low frequencies in the power spectrum (Thompson et al. 2004). For such cases, our twin Gaussian beam model probably overestimates the filtering effect from the interferometer beam. Still, the single and twin Gaussian beam models provide results for two opposite limiting cases (i.e., for the single-dish with minimum filtering at low frequencies and the interferometer with maximum filtering) and allow to specify a range for the dispersion analysis output parameters. For the present cases, the significant uncertainties on some of the other parameters entering the estimates obtained for magnetic field strengths with the DCF equation (e.g., $\rho$ or $\kappa_{\nu}$ ), any error on the output parameters would have a small contribution to the overall uncertainty on any magnetic field strength estimate. But this is probably more a statement on the difficulties encountered when trying to indirectly evaluate magnetic field strengths with techniques not relying on the Zeeman effect. On the other hand, with the future availability of high spatial resolution polarization ALMA data with excellent $u v$-coverage, it is likely that errors on the order of $10 \%$ will become more important when finely characterizing magnetized turbulence in a similar manner as was presented here or through its power spectrum (as in Paper III).

For the TADPOL/CARMA data of Hull et al. (2014) presented in this paper, our analysis yielded a turbulence correlation length $\delta \simeq 19 \mathrm{mpc}$, a ratio of turbulent-to-total magnetic energy $\left\langle B_{\mathrm{t}}^{2}\right\rangle /\left\langle B^{2}\right\rangle \simeq 0.58$, and magnetic field strength $\quad B_{0} \sim 1.1 \mathrm{mG}$ for $\mathrm{W} 3(\mathrm{OH}) ; \quad \delta \simeq 22 \mathrm{mpc}$, $\left\langle B_{\mathrm{t}}^{2}\right\rangle /\left\langle B^{2}\right\rangle \simeq 0.74$, and $B_{0} \sim 0.7 \mathrm{mG}$ for W3 Main; and $\delta \simeq 12 \mathrm{mpc},\left\langle B_{\mathrm{t}}^{2}\right\rangle /\left\langle B^{2}\right\rangle \simeq 0.70$, and $B_{0} \sim 1.2 \mathrm{mG}$ for DR21 $(\mathrm{OH})$. These three sources, therefore, appear to have a significant amount of magnetic energy in the form of turbulence. Finally, our estimates for the turbulence correlation length and magnetic field strengths are consistent with corresponding values obtained from other sources, sometimes obtained with different techniques.

M.H.'s research is funded through the NSERC Discovery Grant, Canada Research Chair, Canada Foundation for Innovation, Ontario Innovation Trust, and Western's Academic Development Fund programs. CARMA development and operations were funded by the National Science Foundation and the CARMA partner universities.

\section{APPENDIX \\ DATA ANALYSIS}

Given the angle difference between a pair of data points separated by $\ell_{i j} \equiv\left|\boldsymbol{r}_{i}-\boldsymbol{r}_{j}\right|$

$$
\Delta \Phi_{i j}=\Phi_{i}-\Phi_{j}
$$

we calculate the mean $\left\langle\cos \left(\Delta \Phi_{i j}\right)\right\rangle_{k}$ from the data for $\left(\ell_{k}-\Delta \ell / 2\right) \leqslant \ell_{i j}<\left(\ell_{k}+\Delta \ell / 2\right)$, with $\ell_{k}=k \Delta \ell$ an integer multiple of the grid spacing $\Delta \ell=0$ ". 25 . This function is then corrected for measurement uncertainties according to

$$
\left\langle\cos \left(\Delta \Phi_{i j}\right)\right\rangle_{k, 0} \simeq \frac{\left\langle\cos \left(\Delta \Phi_{i j}\right)\right\rangle_{k}}{1-\frac{1}{2}\left\langle\sigma^{2}\left(\Delta \Phi_{i j}\right)\right\rangle_{k}},
$$

where the uncertainty on $\Delta \Phi_{i j}$ is given by

$$
\sigma^{2}\left(\Delta \Phi_{i j}\right) \simeq \sigma^{2}\left(\Phi_{i}\right)+\sigma^{2}\left(\Phi_{j}\right)-2 \sigma\left(\Phi_{i}\right) \sigma\left(\Phi_{j}\right) e^{-\ell_{i j}^{2} / 4 W_{1}^{2}}
$$

and $\sigma^{2}\left(\Phi_{i}\right)$ is the uncertainty on $\Phi_{i}$. Equation (29) thus takes into account that pairs of data points will be correlated when separated by approximately less than the telescope beam. From this, the measurement uncertainties for the dispersion function 
$1-\left\langle\cos \left(\Delta \Phi_{i j}\right)\right\rangle_{k, 0}$ are determined through

$$
\begin{aligned}
\sigma^{2}[ & \left.\left\langle\cos \left(\Delta \Phi_{i j}\right)\right\rangle_{k, 0}\right] \\
= & \left\langle\sin \left(\Delta \Phi_{i j}\right)\right\rangle_{k}^{2}\left[\left\langle\sigma^{2}\left(\Delta \Phi_{i j}\right)\right\rangle_{k}+\left\langle\left(\Delta \Phi_{i j}\right)^{2}\right\rangle_{k}\right] \\
& +\left[\frac{3}{4}\left\langle\cos \left(\Delta \Phi_{i j}\right)\right\rangle_{k}^{2}-\left\langle\sin \left(\Delta \Phi_{i j}\right)\right\rangle_{k}^{2}\right] \\
& \times\left[\left\langle\sigma^{2}\left(\Delta \Phi_{i j}\right)\right\rangle_{k}+\left\langle\left(\Delta \Phi_{i j}\right)^{2}\right\rangle_{k}\right]^{2},
\end{aligned}
$$

for all $\left(\ell_{k}-\Delta \ell / 2\right) \leqslant \ell_{i j}<\left(\ell_{k}+\Delta \ell / 2\right)$. Although this analysis follows similar presentations found in Houde et al. (2009, 2013), Equation (30) was augmented to better account for the different sources of uncertainty.

\section{REFERENCES}

Beck, R., Berkhuijsen, E. M., \& Uyanıker, B. 1999, in Plasma Turbulence and Energetic Particles in Astrophysics, ed. M. Otrowski, \& R. Schlickheiser (Kraków: Obs. Astron. Univ. Jagiellongski), 5

Chandrasekhar, S., \& Fermi, E. 1953, ApJ, 118, 113

Chapman, N. L., Goldsmith, P. F., Pineda, J. L., et al. 2011, ApJ, 741, 21

Chini, R., Reipurth, B., Sievers, A., et al. 1997, A\&A, 325, 542

Chitsazzadeh, S., Houde, M., Hildebrand, R. H., \& Vaillancourt, J. E. 2012, ApJ, 749, 45 (Paper IV)

Crutcher, R. M., Troland, T. H., Lazareff, B., Paubert, G., \& Kazès, I. 1999, ApJL, 514, L121

Davis, L., Jr. 1951, PhRv, 81, 890

Dotson, J. L. 1996, ApJ, 470, 566

Dowell, C. D., Hildebrand, R. E., Schleuning, D. A., et al. 1998, ApJ, 504, 588

Falceta-Gonçalves, D., Lazarian, A., \& Kowal, G. 2008, ApJ, 679, 537

Falgarone, E., Troland, T. H., Crutcher, R. M., \& Paubert, G. 2008, A\&A, 487, 247
Fletcher, A., Beck, R., Shukurov, A., Berkhuijsen, E. M., \& Horellou, C. 2011, MNRAS, 412, 2396

Franco, G. A. P., Alves, F. O., \& Girart, J. M. 2010, ApJ, 723, 146

Frisch, U. 1995, Turbulence: The Legacy of A. N. Kolmogorov (Cambridge: Cambridge Univ. Press)

Girart, J. M., Frau, P., Zhang, Q., et al. 2013, ApJ, 772, 69

Goldreich, P., \& Sridhar, S. 1995, ApJ, 438, 763

Hachisuka, K., Brunthaler, A., Menten, K. M., et al. 2006, ApJ, 645, 337

Heiles, C. 1997, ApJS, 111, 245

Hezareh, T., Csengeri, T., Houde, M., Herpin, F., \& Bontemps, S. 2014 MNRAS, 438, 663

Hezareh, T., Houde, M., McCoey, C., \& Li, H. 2010, ApJ, 720, 603

Hildebrand, R. H., Kirby, L., Dotson, J. L., Houde, M., \& Vaillancourt, J. E. 2009, ApJ, 696, 567 (Paper I)

Houde, M., Bastien, P., Peng, R., Phillips, T. G., \& Yoshida, H. 2000a, ApJ, 536,857

Houde, M., Fletcher, A., Beck, R., et al. 2013, ApJ, 766, 49 (Paper V)

Houde, M., Peng, R., Phillips, T. G., Bastien, P., \& Yoshida, H. 2000b, ApJ, 537,245

Houde, M., Phillips, T. G., Bastien, P., Peng, R., \& Yoshida, H. 2001, ApJ, 547,311

Houde, M., Rao, R., Vaillancourt, J. E., \& Hildebrand, R. H. 2011, ApJ, 733 , 109 (Paper III)

Houde, M., Vaillancourt, J. E., Hildebrand, R. H., Chitsazzadeh, S., \& Kirby, L. 2009, ApJ, 706, 1504 (Paper II)

Hull, C. L. H., Plambeck, R. L., Kwon, W., et al. 2014, ApJS, 213, 13

Kobulnicky, H. A., Molnar, L. A., \& Jones, T. J. 1994, ApJ, 107, 1433

Li, H., Attard, M., Dowell, C. D., et al. 2006, Proc. SPIE, 6275, 62751H

Li, H., Dowell, C. D., Kirby, L., Novak, G., \& Vaillancourt, J. E. 2008, ApOpt, 47,422

Li, H., \& Houde, M. 2008, ApJ, 677, 1151

Myers, P. C., \& Goodman, A. A. 1991, ApJ, 373, 509

Novak, G., Chuss, D. T., Davidson, J. A., et al. 2004, Proc. SPIE, 5498, 278

Planck Collaboration Int. XXXV 2016, A\&A, 586, A138

Thompson, A. R., Moran, J. M., \& Swenson, G. W., Jr 2004, Interferometry and Synthesis in Radio Astronomy (Weinheim: Wiley-VCH) 